\title{
Wireless Sensor Network structure for Ground water Well's Field in Karbala City
}

\author{
Hiba A. Tarish \\ University of Information \\ Technology and Communications
}

Baghdad, Iraq

\author{
Mahmoud S. Mahdi, PhD \\ Civil Engineering Department \\ Muayad Sadik Croock, PhD \\ Computer Engineering Department \\ University of Technology Baghdad, University of Technology Baghdad, \\ Iraq
}

\begin{abstract}
Recently, the water resource becomes the most important conditions for living in safe. This is due to the shortage in groundwater, river resources and rain level. This project design a complete sensor network structure to monitor the water levels and water qulity of the considered water wellfield and the consumed electrical power of the installed pump. The utilized sensors use the energy saving techniques and allocate the controller sensor to work as a PAN coordinator. The designed structure has been implemented and tested using MIXIM framework under the environment of OMNET++ simulator. The obtained results from the simulator appear the efficient of the system in monitoring the wells factors.
\end{abstract}

\section{Keywords}

wireless sensor network, wells field, OMNET++.

\section{INTRODUCTION}

Water is one of nature's most valuable resource and fresh water supplies are pushed to the brink of non-renewability by the growing demands on them, [1]. Groundwater resource management is an important issue, especially with regard to agricultural potential [2]. Therefore, it is essential to presents a monitoring system for groundwater wells using wireless sensors and preparation of the control system that has updated information. This is to monitor the critical parameter that affects the groundwater, such as wells water level, EC, consumed power, providing a mechanism to build pumps operation system. For minimizing the consumed operation power and supplying the required water demand.

In [3], groundwater monitoring system has been proposed using wireless pressure sensor network. This is to offer an access to the ground water information by researcher. In [4] authors proposed an irrigation management system based on ZigBee technology. The study showed the suitability of ZigBee for precision agriculture due to its low power consumption, cost and data rate of operation which meet Precision Agriculture (PA) requirements. In [5], real-time monitoring system of groundwater in Korea has been proposed as a mini- review. This is to introduce a continues monitoring of the current status of the ground water networks that can be works in Korea in which some suggestions were presented to enhance the situation of current control and decision making in Korea ground water.
In this paper, a model of wireless sensor network structure is proposed to monitor the essential parameters in the water field of a considered site in Karbala, Iraq. In this structure, a PAN coordinator nodes have been used to control the data flow between the sensor nodes to reduce the periodic time in this network.

\section{THE DESCRIPTION OF THE PROPOSED SYSTEM}

In this section, the proposed system is explained in subsection to ease the reading influence: .

\subsection{Structure of Wireless Sensor Network (WSN)}

WSN uses the IEEE 802.15 .4 network standard for PAN's nodes. In addition, the physical layer adopts to operating in 2.4 GHZ rate of data, it's the limitation band low data rates are suitable for environmental monitoring application like agriculture [6]. Based on the network structure, the WSN can be classify as flat (data centric), hierarchical (cluster-based) and location based (geographical) protocols. These protocols can minimize the total of message passing in efficient way [7] and [8]. The star topology is the best choice for the irrigation applications as it is suitable to small networks in low density of nodes deployment [9]

The model of proposed system is shown in Figure (1). It explains the distribution of wells amongst the groundwater field. In addition, the pipes as well as the pumps on these well. The data is transmitted to the control station in wireless techniques throughout the sink (PAN) nodes. The sensor nodes are classified into the following: the main controller node in this network is PAN coordinator, the (FFDs) node supports the data movement and routing by connecting verity type of networks constriction then forwarding the received messages from the end node until reaching the PAN coordinator. Every node receives a massages coming from all wells in the farm for more processing. In order to manage the traffic model, the network can be classified into: proactive (time-driven model), reactive (exception-driven model), and hybrid network. The sensor nodes in the proactive network [10] and [11]. The hybrid network is efficient in agricultural application due to limited time and fast response to the environmental changes provided from the value of the parameters of sensor [12] Table I shows the important parameters of the utilized sensor network [6]. 


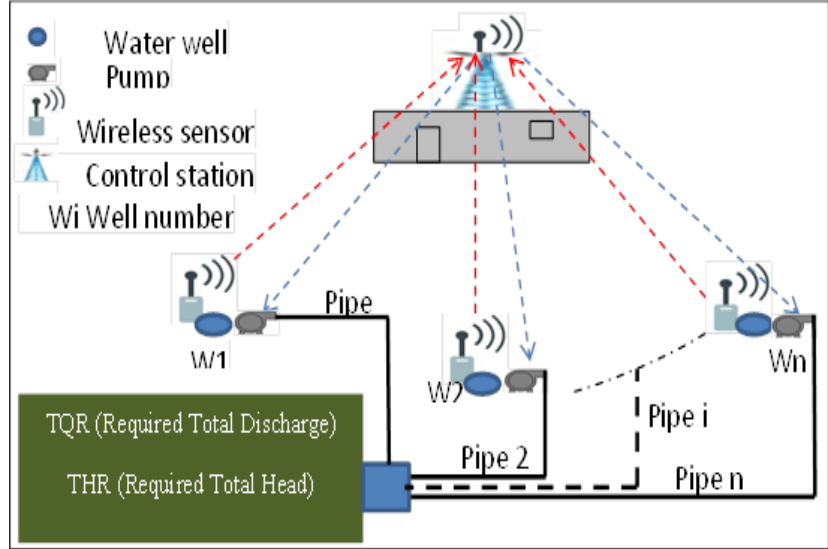

Fig. (1) Structure of WSN based model for well-field operation

Table I NIC specifications

\begin{tabular}{|c|c|}
\hline Parameter & Value \\
\hline Maximum transmission power[mW] & 1.1 \\
\hline Sensitivity[dBm] & -100 \\
\hline Modulation type & MSK \\
\hline Use thermal noise & True \\
\hline Use propagation delay & True \\
\hline Carrier frequency (GHz) & 2.4 \\
\hline Analogue model type & Simple path loss \\
\hline MAC Protocol & CSMA 802.15.4 \\
\hline
\end{tabular}

\subsection{Groundwater Well- Field}

According to agriculture applications, there is a limited distance between the considered nodes [12]. Therefore, the distance between the nodes in the designed structure is reduced to minimum in which the system works efficiently. In proposed system, three farms (A,B, and C) with complete area of 1000 Dunam. The field includes 24 sensor nodes distributed over 24 wells. These wells are distributed into (4) wells in farm A. 16 wells in farm B and (4) wells in C.

\subsection{WSN Node Communication}

WSN includes different types of nodes that have many parameters that can describe the behavior of nodes in terms of characteristics through the simulation processes, such as NICs parameter, channel characteristics or specification. The considered channel specifications is shown in Table II

Table II channel specification

\begin{tabular}{|c|c|}
\hline Parameter & Value \\
\hline Minimum path loss coefficient(a) & 2.5 \\
\hline Minimum signal attenuation threshold (dBm) & -100 \\
\hline
\end{tabular}

The end nods collects the data of wells and then send these data to the head or main node that gathers the received messages to be sent to the RFD node. The received data in RFD is sent to the PAN coordinator nodes. The duty of coordinator is the decision making process, for example if the well have maximum level of water , less EC , and generating in less power, the PAN coordinator have the decision of switch $\mathrm{OF} / \mathrm{ON}$ of the best group of wells in each farm.

\section{SIMULATED RESULTS}

The proposed system considers effective tool to management and control the operation of wells in best way. As mentioned earlier, the simulator is OMNET++ and the considered underground well fields is located in Karbala, Iraq.

Figure (2) shows the ability of head nodes to minimize the value of power compering with other end nodes allocated on other wells. It is well explained that the power consuming is reduced with the increasing of the number of nodes due to the ability of selecting the optimal operated nodes in which the system is kept efficient.

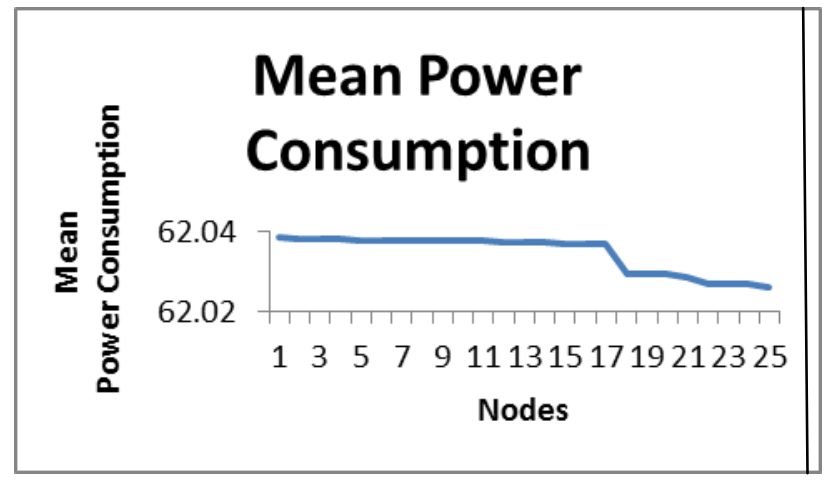

Fig. (2) The mean power consumed vs. number of nodes 
Figure (3) explains the received packet of data for every node. The simulation model is working to reducing number of message that can be sending to the PAN coordinator. The utilized topology can reduce the data traffic to manage the network in easer way.

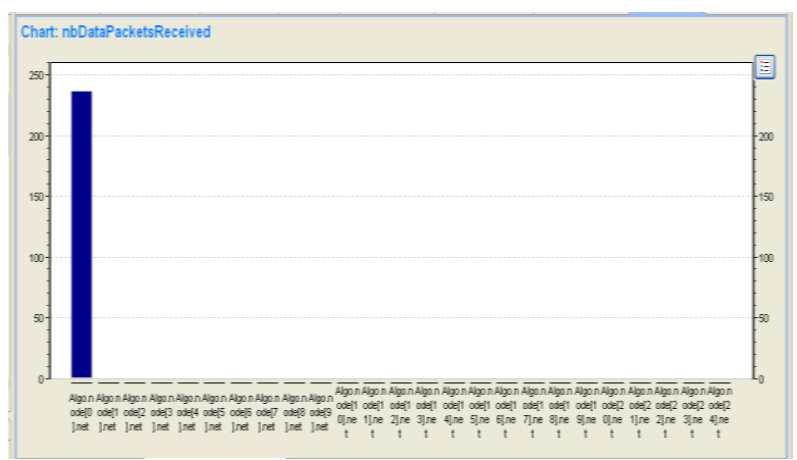

Fig. (3) Number of received packets

The interference between frames is shown in Figure (4). As mentioned above, the simulator uses IEEE 802.15.4 standard, (CSMA/ CA) carrier sense multiple access with collisions avoidance (CSMA/CA) protocol

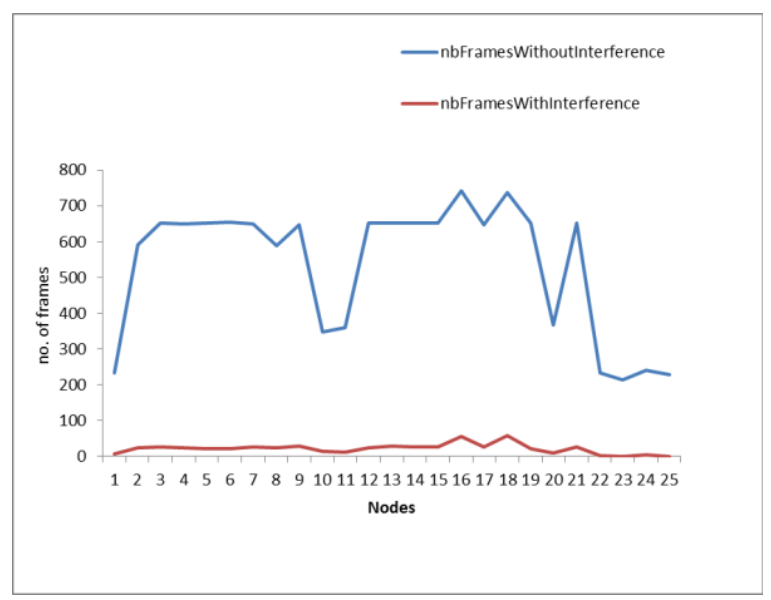

Fig. (4) Interference between frames

Additional, it is important to investigate the Back off duration of the entire nodes. It refers to the waiting time while the node is trying to transmit a letter when the transmission medium is busy. The use of CSMA/CA based protocols tackles this problem by resending the same message when the waiting time is finished. Figure (5) shows the number of back offs per node, and Figure (6) shows the back offs durations.

\section{CONCLUSIONS}

A designed wireless sensor network structure has been proposed. This sensors are distributed amongst the wellFieleds in agricultural site; Karbala city, Iraq. The structure monitored the important parameters of each well individually and then collected the overall data for processing and decision making. A complete analysis is provided for the sensor nodes in terms of power consumption and other factors. The considered site included three farms with 24 water wells distributed with 4, 4 and 16 wells amongst the farms. The simulated results show the efficiency of the monitoring procedure of the watar wells. The factors of consumed power, package massage transmission and interface between nodes of sensor network have been studied well. This study showed the efficient of operation in terms of monitoring. It is also noted that the accrued problems of interference have been solved automatically throughout the network operation. OMNET++ simulator has been used for implementing the proposed system.as future work, the proposed project can be implemented in hardware design.

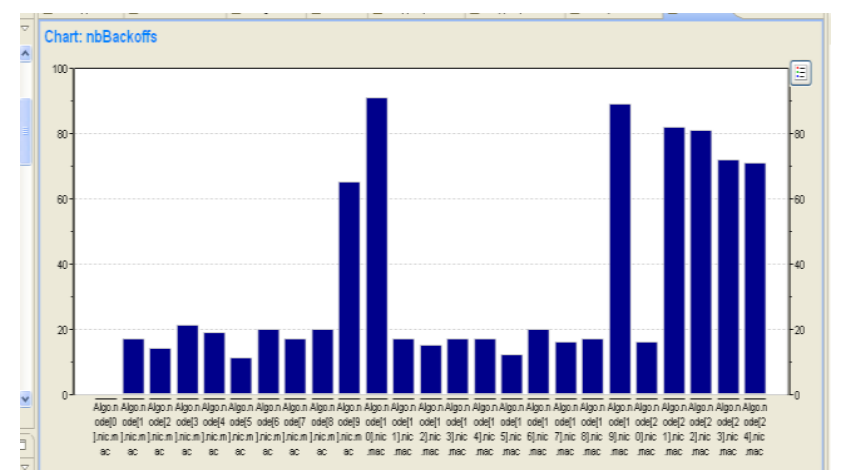

Fig. (5) Number of back-off per node

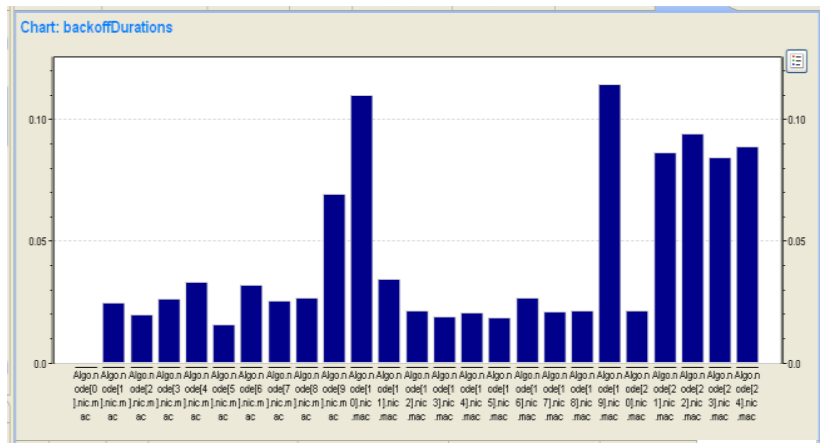

Fig. (6) Back-off durations

\section{REFERENCES}

[1] David Tood , Larry W.Mays, GroundWater Hydrology , third Edition.

[2] Jassas H. and Merkel B., "Estimating Groundwater Recharge in the Semiarid Al-Khazir Gomal Basin, North Iraq”, Water Journal, Vol. 6(8), pp. 2467-2481. (2014)

[3] Srikanth Anumalla , and David C. Gosselin , " Ground Water Monitoring using Smart Sensors" IEEE International Conference on Electro Information Technology, 2005.

[4] M. Mafuta, M. Zennaro, A. Bagula, G. Ault, H. Gombachika and T. Chadza, "Successful Deployment of a Wireless Sensor Network for Precision Agriculture in Malawi”, International Journal of Distributed Sensor Networks, Vol.2013, PP.1-13, 2013

[5] Jin-Yong Lee , and Kideok D. Kwon, "Current Status of Groundwater Monitoring Networks in Korea "article ,water http://creativecommons.org/licenses/by/4.0/ 2016.

[6] M. Popa, O. Prostean and A.S. Popa, "A Classification of Solutions for the Energy Limitation in Wireless Sensor Networks", IEEE 9th International Conference on Computational Cybernetics, PP.293-297, 2013.

[7] P. Tyagi and S. Jain, "Comparative Study of Routing Protocols in Wireless Sensor Network", International Journal of Advanced Research in Computer Science and Software Engineering, Vol.2, No.9, PP.134ᄀ140, 2012.

[8] S. Ahirwar and P. Tanwar, "A Review of Power Optimization Strategies in Wireless Sensor Network", 
International Journal of Emerging Technology and Advanced Engineering (IJETAE), Vol.3, No.10, PP.212218, 2013.

[9] P. Jurmk, "Real-time Communication over Cluster-tree Wireless Sensor Networks", Ph.D. thesis, Czech Technical University in Prague, 2010.

[10] K. Kondaiah, R.B. Babu and S. Umar, "A Study on Routing Protocols in Wireless Sensor Networks", International Journal of Computer Science Engineering and Technology (IJCSET), Vol.3, No.3, PP.94-98, 2013.

[11] S. E. Díaz, J. C. Pérez, A. C. Mateos, M.-C. Marinescu, and B. B. Guerra, Computers and Electronics in Agriculture 76, 252, (2011).

[12] S. M. Abd El-kader and B. M. M. El-Basioni, "Precision farming solution in Egypt using the wireless sensor network technology", Egyptian Informatics Journal, Vol.14, No.3, PP.221-233, 2013 